\title{
ASYMPTOTIC ANALYSIS OF EIGENVALUE-BASED BLIND SPECTRUM SENSING TECHNIQUES
}

\author{
Symeon Chatzinotas, Shree Krishna Sharma and Björn Ottersten
}

\author{
SnT - securityandtrust.lu, University of Luxembourg \\ Email:\{symeon.chatzinotas, shree.sharma,bjorn.ottersten\}@uni.lu
}

\begin{abstract}
Herein, we consider asymptotic performance analysis of eigenvalue-based blind Spectrum Sensing (SS) techniques for large-scale Cognitive Radio (CR) networks using Random Matrix Theory (RMT). Different methods such as Scaled Largest Value (SLE), Standard Condition Number (SCN), John's detection and Spherical Test (ST) based detection are considered. The asymptotic sensing bounds for John's detection and ST based detection techniques are derived under a noise only hypothesis for sensing the presence of Primary Users (PUs). These asymptotic bounds are then used as thresholds for the SS decision and their performance is compared with other techniques in terms of probability of correct detection under both hypotheses. It is noted that the SLE detector is the best for a range of scenarios, followed by JD, SCN, ST. Furthermore, it is shown that noise correlation significantly degrades the performance of ST and JD detectors in practical scenarios.
\end{abstract}

Index Terms - Spectrum Sensing, Asymptotic Analysis, Cognitive Radio, Random Matrix theory

\section{INTRODUCTION}

The opportunistic access of idle primary spectrum by other systems without affecting the performance of primary owners has been considered as a promising solution to improve the spectral efficiency of future wireless networks. Several dimensions such as frequency, space, area, polarization $[1,2]$ and angular dimension of the signal space [3] have been considered in the literature [4] towards identifying and exploring idle spectrum holes. In this direction, Spectrum Sensing (SS) has been considered a key component for dynamic spectrum access required by a Cognitive Radio (CR) system. Several $\mathrm{SS}$ techniques have been proposed in the literature for sensing the presence of a Primary User (PU) [4]. Furthermore, several diversity enhancement techniques such as multi-antenna, cooperative and oversampled techniques have been introduced in order to enhance the SS efficiency in wireless fading channels $[5,6,7]$. These methods mostly consider the statistics of the eigenvalues of the received signal's covariance matrix using recent results from Random Matrix Theory (RMT). The main advantage of eigenvalue based SS techniques in practical scenarios is that it does not require any prior information of the PU's signal and it outperforms Energy Detection (ED) techniques, especially in the presence of noise covariance uncertainty [5].

1) Related Work: Several eigenvalue based algorithms have been proposed in the literature $[5,9,10,11,12,6,7$, 13, 14, 15] exploiting RMT methods. These techniques can be categorized into Signal Condition Number (SCN) based [5, 10, 14, 6], Largest Eigenvalue (LE) based [16, 17, 11] and Scaled Largest Eigenvalue (SLE) based [18, 19, 20]. Furthermore, several SCN based techniques such as asymptotic [9], semi-asymptotic [5] and ratio based techniques have been proposed utilizing the properties of the eigenvalues of random Wishart matrices. The authors in [9] use the Marcenko-Pastur (MP) law to test a binary hypothesis assuming the presence of white noise. In [5], semi-asymptotic MME and EME algorithms for SS have been proposed using the combination of the MP based and Tracy-Widom (TW) distribution based approaches and in [10], ratio based technique has been proposed using the Tracy-Widom Curtiss (TWC) distribution. A new detection threshold has been proposed in [14] for improved sensing performance in the presence of noise correlation. In addition, a Signal to Noise Ratio (SNR) estimation technique has been proposed to estimate the SNR of the PU signal in the presence of correlated noise. In [21], non-asymptotic behavior of eigenvalues of random matrices has been considered using the spectral properties of random sub-Gaussian matrices of fixed dimensions. A cooperative SS algorithm using double eigenvalue thresholds has been proposed in [22], which considers two maximum eigenvalues for the noise only and the signal plus noise cases.

Recently, the distribution of the SCN of Wishart matrices has been considered in signal detection for a CR [23]. In this context, two types of condition numbers i.e., SCN (the ratio of the maximum to the minimum eigenvalue) and Demmel Condition Number (DCN) (the ratio of the matrix trace to the minimum eigenvalue) have been considered. These two metrics provide a measure of rank deficiency of a matrix and their statistical properties can be used in a variety of applications. In [23], a general framework for the Cumulative Distribution Function (CDF) of the SCN of different classes of Wishart 
matrices has been presented. In [24], the exact distribution of the DCN for random matrices with arbitrary dimensions has been presented. In [25], analytic expressions for the Probability Density Function (PDF) and CDF of the ratio of the largest eigenvalue to the trace of complex Wishart matrices with arbitrary dimensions have been derived.

In addition to the above mentioned methods, Spherical Test (ST) based detection [13, 26, 27, 28] and John's detection [13] have also been considered in the literature. In [28], a new sphericity test statistic has been proposed to detect closely spaced sources with improved resolution and to detect sources with lower SNRs. In [13], ST detector and John's detector have been used for SS purposes in the presence of multiple PUs considering white noise. However, the effect of noise correlation on the performance of these detectors has not been addressed in [13]. In practical scenarios, noise correlation may arise due to imperfections in filtering and oversampling in the receiver [5].

2) Contributions: In this paper, we study the performance of different eigenvalue based blind SS techniques using asymptotic analysis. In the literature, asymptotic bounds for LE, SLE and SCN methods have been proposed but the asymptotic thresholds for ST detector and John's detector are still missing. In this direction, the main contribution of this paper is the derivation of asymptotic thresholds for ST and John's detectors. Although finite approximations have been considered in the literature [13] for these techniques, the expressions for thresholds contain involved functions and are complex to evaluate. In case of finite analysis, the threshold is determined based on the probability of false alarm (pfa) and choosing a correct pfa rate for a particular system may be problematic in practical scenarios. On the other hand, asymptotic thresholds are deterministic ("hardening" effect) and allow for simpler sensing decisions under both hypotheses [14]. Furthermore, they may provide more accurate performance analysis of a sensing technique in presence of correlation. In this direction, another contribution of this paper is to study the effect of noise correlation on the performance of the considered SS techniques.

The remainder of this paper is structured as follows: Section 2 provides the signal and channel model. Section 3 presents the test statistics and decision methods for considered eigenvalue based SS techniques. Section 4 presents the theoretical analysis of ST and John's detection methods under noise only case. Section 5 compares the sensing performance of different asymptotic methods with the help of numerical results. Section 6 concludes the paper.

3) Notation Throughout the formulations of this paper, boldface upper and lower case letters are used to denote matrices and vectors respectively, $\mathbb{E}[\cdot]$ denotes the expectation, $(\cdot)^{T}$ denotes the transpose matrix, $(\cdot)^{H}$ denotes the conjugate transpose matrix, $\operatorname{tr}\{\cdot\}$ represents the trace of a matrix, $\lambda_{\max }(\cdot)$ and $\lambda_{\min }(\cdot)$ denote the maximum eigenvalue and the minimum eigenvalue of a matrix respectively. Finally, $(\cdot)^{\infty}$ denotes asymptotic deterministic values of random metrics.

\section{SIGNAL MODEL}

We consider a large CR network with $M$ cooperating nodes ${ }^{1}$ and each node is equipped with a single antenna. The factor $M$ corresponds to the number of antennas in single-node multi-antenna assisted SS [18]. Let $N$ be the number of observations collected by each CR node in the time duration of $\tau$. We consider a generic signal model assuming the presence of multiple PUs, while the signal model for the case of single PU can be considered as a specific case.

We assume that channel remains constant during the period of sensing ${ }^{2}$ and the transmitted PU symbols are i.i.d. complex circularly symmetric (c.c.s.) Gaussian symbols. A single observation of the $M \times 1$ received signal $y$ in presence of $K$ PUs can be written as:

$$
\mathbf{y}=\sum_{i=1}^{K} \mathbf{h}_{i} s_{i}+\mathbf{z}=\mathbf{H} \mathbf{s}+\mathbf{z}
$$

where $\mathbf{s}$ is $K \times 1$ transmitted signal i.e., $\mathbf{s}=\left[s_{1}, s_{2}, \ldots, s_{K}\right]^{T}$, with $s_{i}$ being a Gaussian symbol with power $p_{i}=\mathbb{E}\left[s_{i}^{2}\right]$. The $M \times K$ channel matrix $\mathbf{H}$ includes the channel coefficients between PUs and $M$ receive nodes i.e., $\mathbf{H}=\left[\mathbf{h}_{1} \mathbf{h}_{2} \ldots \mathbf{h}_{K}\right]$, $\mathbf{z}$ is $M \times 1$ Gaussian noise vector with zero mean and variance $\sigma^{2}$. After collecting $N$ samples for each receiving node, the $M \times N$ received signal matrix $\mathbf{Y}$ can be written as:

$$
\mathbf{Y}=\left[\mathbf{y}_{1}, \mathbf{y}_{2} \ldots \mathbf{y}_{N}\right]=\left[\begin{array}{cccc}
y_{1}(1) & y_{1}(2) & \ldots & y_{1}(N) \\
y_{2}(1) & y_{2}(2) & \ldots & y_{2}(N) \\
\vdots & \vdots & \ddots & \vdots \\
y_{M}(1) & y_{M}(2) & \ldots & y_{M}(N)
\end{array}\right]
$$

Let us denote the hypotheses of the presence and absence of the PU signal by $\mathbb{H}_{1}$ and $\mathbb{H}_{0}$ respectively. The binary hypothesis testing problem for deciding the presence of a PU signal can be written as:

$$
\mathbb{H}_{0}: \mathbf{Y}=\mathbf{Z}, \quad \mathbb{H}_{1}: \mathbf{Y}=\mathbf{H S}+\mathbf{Z}
$$

where $\mathbf{S}$ is the $K \times N$ transmitted signal and $\mathbf{Z}$ is the $M \times N$ Gaussian noise. Let us define sample covariance matrices of received signal and noise as: $\mathbf{R}_{\mathbf{Y}}(N)=\frac{1}{N} \mathbf{Y} \mathbf{Y}^{H}$ and $\mathbf{R}_{\mathbf{Z}}(N)=\frac{1}{N} \mathbf{Z} \mathbf{Z}^{H}$. Under noise only hypothesis, the sample covariance matrix of the received signal becomes equal to sample covariance matrix of noise i.e., $\mathbf{R}_{\mathbf{Y}}(N)=\mathbf{R}_{\mathbf{Z}}(N)$. It can be noted that since $\mathbf{Z} \sim \mathcal{C N}(0, \mathbf{I}), \mathbf{Z} \mathbf{Z}^{H}$ follows an uncorrelated Wishart distribution [5] i.e., $\mathbf{Z} \mathbf{Z}^{H} \sim \mathcal{W}_{M}(\boldsymbol{\Sigma}, N)$, where $\boldsymbol{\Sigma}=\frac{\mathbb{E}\left[\mathbf{Z Z}^{H}\right]}{N}$. Let us denote the ordered eigenvalues of $\mathbf{R}_{\mathbf{Y}}$ by $\lambda_{1} \geq \lambda_{2} \geq \lambda_{3} \geq \cdots \geq \lambda_{M}$. It can be noted that since

\footnotetext{
${ }^{1}$ In practice, the cooperating nodes can be connected to a centralized decision center with a backhaul link.

${ }^{2}$ This assumption does not affect the asymptotic thresholds since they are based on $\mathbb{H}_{0}$ hypothesis statistics.
} 
our sensing threshold only depends on the $\mathbb{H}_{0}$ hypothesis i.e., on the eigenvalue properties of $\mathbf{R}_{\mathbf{Z}}$, various channel and signal types can be considered under the considered framework.

\section{TEST STATISTICS}

1) Scaled Largest Eigenvalue Technique: The decision for SLE method can be made on the basis of following binary hypothesis testing [20]:

$$
\text { decision }= \begin{cases}\mathbb{H}_{0}, & \text { if } \frac{\lambda_{\max }\left(\mathbf{R}_{\mathbf{Y}}(N)\right)}{\frac{1}{M} \operatorname{tr}\left\{\mathbf{R}_{\mathbf{Y}}(N)\right\}} \leq T_{S L E}^{\infty} \\ \mathbb{H}_{1}, & \text { otherwise }\end{cases}
$$

where $T_{S L E}^{\infty}$ is the asymptotic threshold for the SLE method.

2) SCN based Technique: The decision for SCN based technique can be made in the following way [15]:

$$
\text { decision }= \begin{cases}\mathbb{H}_{0}, & \text { if } \frac{\lambda_{\max }\left(\mathbf{R}_{\mathbf{Y}}(\mathrm{N})\right)}{\lambda_{\min }\left(\mathbf{R}_{\mathbf{Y}}(\mathrm{N})\right)} \leq T_{S C N}^{\infty} \\ \mathbb{H}_{1}, & \text { otherwise }\end{cases}
$$

where $T_{S C N}^{\infty}$ is the asymptotic threshold for the SCN method.

3) Spherical Test Method: The test statistic for this method is calculated as the ratio of the geometric and arithmetic mean of all eigenvalues [13]:

$$
T_{S T}=\frac{\left(\operatorname{det}\left(\mathbf{R}_{\mathbf{Y}}(N)\right)\right)^{1 / M}}{\frac{1}{M} \operatorname{tr}\left(\mathbf{R}_{\mathbf{Y}}(N)\right)}=\frac{\left(\prod_{i=1}^{M} \lambda_{i}\right)^{1 / M}}{\frac{1}{M} \Sigma_{i=1}^{M} \lambda_{i}}
$$

The binary hypothesis testing based on this method can be expressed as:

$$
\text { decision }= \begin{cases}\mathbb{H}_{0}, & \text { if } T_{S T} \geq T_{S T}^{\infty} \\ \mathbb{H}_{1}, & \text { otherwise }\end{cases}
$$

where $T_{S T}^{\infty}$ represents the asymptotic threshold for the ST method.

4) John's Detection Method: The test statistic for this method is given as the ratio of the quadratic mean over the arithmetic mean of all eigenvalues [13] i.e., $T_{J}=\frac{\sqrt{\sum_{i=1}^{M} \lambda_{i}^{2}}}{\sum_{i=1}^{M} \lambda_{i}}$. The binary hypothesis testing based on this method can be expressed as:

$$
\text { decision }= \begin{cases}\mathbb{H}_{0}, & \text { if } T_{J} \leq T_{J}^{\infty} \\ \mathbb{H}_{1}, & \text { otherwise }\end{cases}
$$

where $T_{J}^{\infty}$ represents the asymptotic threshold for JD detector.

\section{ASYMPTOTIC ANALYSIS UNDER $\mathbb{H}_{0}$}

The asymptotic analysis in the aforementioned problem is based on the Marcenko-Pastur law which we mention here for completeness.
Theorem 4.1 [8] Consider an $M \times N$ matrix $\mathbf{F}$ whose entries are independent zero-mean complex (or real) random variables with variance $\frac{1}{N}$ and fourth moments of order $O\left(\frac{1}{N^{2}}\right)$. As $M, N \rightarrow \infty$ with $\frac{N}{M} \rightarrow \beta$, the empirical distribution of the eigenvalues of $\frac{1}{M} \mathbf{F} \mathbf{F}^{H}$ converges almost surely to a non random limiting distribution with density given by;

$$
f_{\beta}(\lambda)=(1-\beta)^{+} \delta(\lambda)+\frac{\sqrt{(\lambda-a)^{+}(b-\lambda)^{+}}}{2 \pi \beta \lambda}
$$

where $a=(1-\sqrt{\beta})^{2}, b=(1+\sqrt{\beta})^{2}, \delta($.$) is a Dirac delta$ function and $(1-\beta)^{+} \delta(\lambda)$ represents the cardinality of zero eigenvalues which can occur if $M>N$. The parameters a and $b$ define the support of the distribution and correspond to $\lambda_{\min }^{\infty}$ and $\lambda_{\max }^{\infty}$ respectively and the ratio b/a defines the SCN of $\frac{1}{M} \mathbf{F F}^{H}$. The above limiting distribution is the MP law with ratio index $\beta$.

Lemma 4.1 [8] The empirical distribution of $\mathbf{F F}^{H}$, with $\mathbf{F}$ as in Theorem 4.1, converges almost surely to a nonrandom limiting distribution with density (8) whose moments are given by;

$$
\lim _{M, N \rightarrow \infty} \frac{1}{M} \operatorname{tr}\left\{\left(\mathbf{F} \mathbf{F}^{H}\right)^{k}\right\}=\sum_{i=1}^{k} \frac{1}{k}\left(\begin{array}{c}
k \\
i
\end{array}\right)\left(\begin{array}{c}
k \\
i-1
\end{array}\right) \beta^{i}
$$

1) Scaled Largest Value Technique: According to Lemma 4.1, the asymptotic arithmetic mean of the eigenvalues equals:

$$
\lim _{M, N \rightarrow \infty} \frac{1}{M} \operatorname{tr}\left\{\mathbf{R}_{\mathbf{Y}}(N)\right\}=\frac{1}{M} \Sigma_{i=1}^{M} \lambda_{i}=\beta
$$

The largest eigenvalue converges to $(1+\sqrt{\beta})^{2}$ as given by the MP law in Theorem 4.1, and thus the asymptotic SLE threshold converges to: $T_{S L E}^{\infty}=(1+1 / \sqrt{\beta})^{2}$.

2) SCN Based Technique: Based on the above mentioned MP law, the asymptotic SCN based threshold converges to: $T_{S C N}^{\infty}=\frac{(1+\sqrt{\beta})^{2}}{(1-\sqrt{\beta})^{2}}$.

Lemma 4.2 The asymptotic geometric mean of the eigenvalues converges to:

$$
\lim _{M, N \rightarrow \infty}\left(\prod_{i=1}^{M} \lambda_{i}\right)^{1 / M}=\beta \exp (-1-(\beta-1) \log (1-1 / \beta))
$$

Proof: The geometric mean can be rewritten as:

$$
\left(\prod_{i=1}^{M} \lambda_{i}\right)^{1 / M}=\exp \left(\log \left(\prod_{i=1}^{M} \lambda_{i}\right)^{1 / M}\right)=\exp \left(\frac{1}{M} \Sigma_{i=1}^{M} \log \left(\lambda_{i}\right)\right)
$$

The argument of the exponent can be calculated by integrating over the MP distribution:

$$
\lim _{M, N \rightarrow \infty} \frac{1}{M} \sum_{i=1}^{M} \log \left(\lambda_{i}\right)=\int_{a}^{b} \log (x) f_{\beta}(x) d x
$$

The integration result in (10) follows after a series of change of variables $[29,30]$ and Cauchy integration which are omitted due to lack of space. 
3) Spherical Test Method: The asymptotic ST threshold converges to: $T_{S T}^{\infty}=\exp (-1-(\beta-1) \log (1-1 / \beta))$.

4) John's Detection Method: According to Lemma 4.1, the asymptotic quadratic mean of the eigenvalues converges to: $\lim _{M, N \rightarrow \infty} \sqrt{\frac{1}{M} \sum_{i=1}^{M} \lambda_{i}^{2}}=\sqrt{\beta^{2}+\beta}$. The asymptotic JD threshold converges to: $T_{J}^{\infty}=\sqrt{1+1 / \beta}$.

\section{NUMERICAL RESULTS}

1) Performance Metric: The ratio of correct sensing is used as a performance metric to analyze the performance and it is defined as the ratio of number of correct sensing to the number of total considered iterations under both hypotheses. This ratio should be higher than 0.5 which is the ratio for coin-flip decision making. This term can be expressed in terms of $P_{d}$ and $P_{f}$ as: $\left(P_{d}+\left(1-P_{f}\right)\right) / 2$, where $P_{d}$ and $1-P_{f}$ denote the number of correct sensing out of the total considered iterations under $\mathbb{H}_{1}$ and $\mathbb{H}_{0}$ hypotheses respectively.

2) Considering Finite Dimensions: The above mentioned asymptotic thresholds will have non-zero variance while running simulations for finite dimensions. As a result, we have to compensate for this variance in order to ensure that probability of false alarm under hypothesis $\mathbb{H}_{0}$ becomes negligible for large noise variance. Let $\delta_{S T}=\mathbb{E}\left[T_{S T}^{2}\right]$ and $\delta_{J}=\mathbb{E}\left[T_{J}^{2}\right]$ denote the variances for ST and JD detectors ${ }^{3}$ when considering large but finite dimensions, then the thresholds need to be adjusted as: $\bar{T}_{S T}^{\infty}=T_{S T}^{\infty}-\delta_{S T}$ and $\bar{T}_{J}^{\infty}=T_{J}^{\infty}+\delta_{J}$ taking into account the inequalities in Section 3.

3) Results: Figure 1 shows the ratio of correct sensing versus SNR for different techniques with parameters $\beta=$ $10, N=100$ in Rayleigh fading channel assuming the channel remains constant across during the period of sensing. In this simulation settings, the values of $\delta_{J D}$ and $\delta_{S T}$ are considered to be 0.04 and 0.03 respectively. These values were calculated based on CDF curves of the decision statistics numerically. From the result, it can be noted that SLE detector performs the best among other detectors and JD detector performs slightly worse than the SLE detector and better than SCN-based and ST detectors. Furthermore, for $90 \%$ correct sensing, the SLE detector requires almost $3 \mathrm{~dB}$ less SNR as compared to the ST detector, the JD detector requires almost $2 \mathrm{~dB}$ less SNR as compared to the ST detector and the SCNbased detector requires almost $1 \mathrm{~dB}$ less SNR than the ST detector. During simulation, it was observed that the same performance ordering was preserved for multiple user scenario and for Rician fading channel conditions.

To evaluate the performance of considered techniques in presence of noise correlation, noise vector $\mathbf{z}$ was considered to be correlated with $\mathbb{E}\left[\mathbf{z z}^{H}\right]=\mathbf{R}$, which follows a leftsided exponential correlation model with correlation coeffi-

\footnotetext{
${ }^{3}$ No compensation was needed for SCN and SLE since the asymptotic thresholds cleared the support of the random values during finite-size simulations.
}

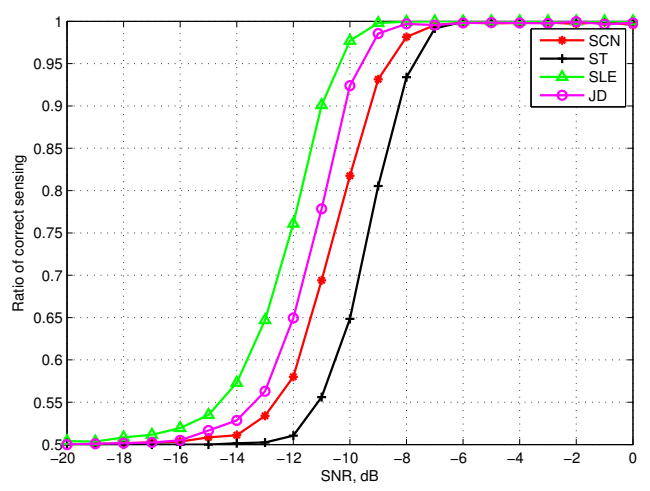

Fig. 1. Ratio of correct sensing versus SNR for different techniques in Rayleigh fading channel $(\beta=10, N=100)$

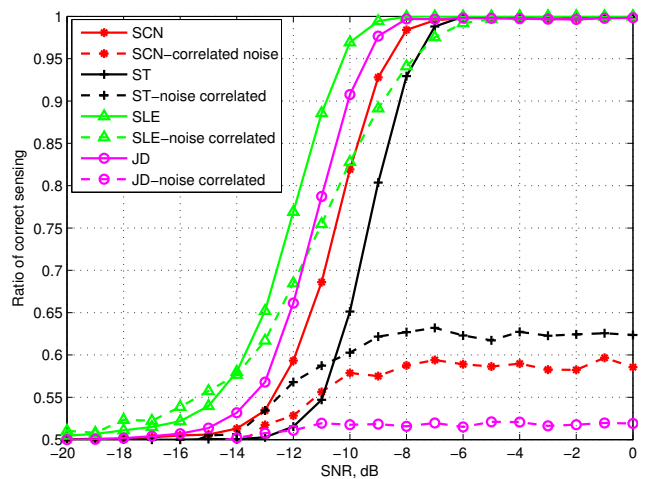

Fig. 2. Ratio of correct sensing versus SNR for different techniques in presence of correlated noise $(\beta=10, N=100, \mathrm{SCN}=2.64)$

cient $\rho=\frac{1-\mathrm{SCN}}{1+\mathrm{SCN}}$ [14]. Simulations were carried out with the parameters $\beta=10, N=100$ and $\mathrm{SCN}=2.64$. Figure 2 shows the effect of noise correlation over different techniques and it can be noted that JD detector has the worst performance of all the techniques and SLE detector is the best in terms of sensing performance in presence of correlated noise. The detailed analysis of noise correlation effect on the SCN-based techniques can be found in [14].

\section{CONCLUSION}

In this paper, an asymptotic analysis was presented for ST and JD detectors under noise only hypothesis. The performance of these techniques has been compared with other techniques in different scenarios. It has been observed that the performance of the SLE detector is the best for a range of scenarios, followed by JD, SCN and ST. Moreover, it can be concluded that the SLE detector is the best in terms of sensing performance in the presence of correlated noise, while the performance of the JD and ST detectors greatly deteriorates in the presence of noise correlation. Finding an analytic methodology for calculating the finite variance $\delta$ for the considered thresholds is an open issue for future work in this field. 


\section{REFERENCES}

[1] S. K. Sharma, S. Chatzinotas, and B. Ottersten, "Exploiting polarization for spectrum sensing in cognitive satcoms," in 7th Int. Conf. CROWNCOM, June 2012.

[2] S. K. Sharma, S. Chatzinotas, and B. Ottersten, "Spectrum sensing in dual polarized fading channels for cognitive satcoms," in IEEE Globecom Conf., Dec. 2012.

[3] E. Tsakalaki, and et al, "Spectrum sensing using single-radio switched-beam antenna systems," in 7th Int. Conf. CROWNCOM, June 2012

[4] T. Yucek and H. Arslan, "A survey of spectrum sensing algorithms for cognitive radio applications," IEEE Commun. Surveys Tutorials, vol. 11, no. 1, pp. 116 -130, quarter 2009.

[5] Y. Zeng and Y. C. Liang, "Eigenvalue-based spectrum sensing algorithms for cognitive radio," IEEE Trans. Commun., vol. 57, no. 6, pp. $1784-1793$, June 2009.

[6] W. Zhang, G. Abreu, M. Inamori, and Y. Sanada, "Spectrum sensing algorithms via finite random matrices," IEEE Trans. Commun., vol. 60, no. 1, pp. 164 -175, Jan. 2012.

[7] A. Kortun, T. Ratnarajah, M. Sellathurai, C. Zhong, and C. B. Papadias, "On the performance of eigenvalue-based cooperative spectrum sensing for cognitive radio," IEEE J. Selected Topics in Signal Process., vol. 5, no. 1, pp. 49 -55, Feb. 2011.

[8] A. M. Tulino and S. Verdu, "Random matrix theory and wireless communications," Foundations and Trends in Commun. and Inf. Th., vol. 1, no. 1, pp. 1-182, 2004.

[9] L.S. Cardoso, M. Debbah, P. Bianchi, and J. Najim, "Cooperative spectrum sensing using random matrix theory," in $3 \mathrm{rd}$ Int. Symp. Wireless Pervasive Computing, May 2008, pp. 334 -338 .

[10] F. Penna, R. Garello, and M. Spirito, "Cooperative spectrum sensing based on the limiting eigenvalue ratio distribution in Wishart matrices," IEEE Commun. Letters, vol. 13, no. 7, pp. $507-509$, July 2009.

[11] A. Taherpour, M. Nasiri-Kenari, and S. Gazor, "Multiple antenna spectrum sensing in cognitive radios," IEEE Trans. Wireless Commun., vol. 9, no. 2, pp. 814 -823, Feb. 2010.

[12] K. Hassan, R. Gautier, I. Dayoub, E. Radoi, and M. Berbineau, "Predicted eigenvalue threshold based spectrum sensing with correlated multiple-antennas," in IEEE 75th VTC Spring, May 2012, pp. $1-5$.

[13] Lu Wei and O. Tirkkonen, "Spectrum sensing in the presence of multiple primary users," IEEE Trans. Commun., vol. 60, no. 5, pp. 1268 -1277, May 2012.

[14] S. K. Sharma, S. Chatzinotas, and B. Ottersten, "Eigenvalue based sensing and SNR estimation for cognitive radio in presence of noise correlation," IEEE Trans. Veh. Techn., accepted, 2013.

[15] S. K. Sharma, S. Chatzinotas, and B. Ottersten, "The Effect of Noise Correlation on Fractional Sampling based Spectrum Sensing," to appear in Proc. IEEE ICC, June 2013.

[16] Y. Zeng, C. L. Koh, and Y. Liang, "Maximum eigenvalue detection: Theory and application," in IEEE ICC, May 2008, pp. $4160-4164$.
[17] L. Wei and O. Tirkkonen, "Cooperative spectrum sensing of ofdm signals using largest eigenvalue distributions," in IEEE 20th Int. Symp. PIMRC, sept. 2009, pp. 2295 -2299.

[18] P. Wang, J. Fang, N. Han, and Hongbin Li, "Multiantennaassisted spectrum sensing for cognitive radio," IEEE Trans. Veh. Techn., vol. 59, no. 4, pp. 1791 -1800, May 2010.

[19] P. Bianchi, M. Debbah, M. Maida, and J. Najim, "Performance of statistical tests for single-source detection using random matrix theory," IEEE Trans. Inf. Th., vol. 57, no. 4, pp. 2400 -2419, April 2011.

[20] L. Wei and O. Tirkkonen, "Analysis of scaled largest eigenvalue based detection for spectrum sensing," in Proc. IEEE ICC, June 2011, pp. 1 -5.

[21] L. Wang, B. Zheng, J. Cui, and W. Yue, "Spectrum sensing using non-asymptotic behavior of eigenvalues," in Int. Conf. Wireless Commun. and Signal Process., Nov. 2011, pp. 1 -5.

[22] K. Cao and Z. Yang, "A novel cooperative spectrum sensing algorithm based on random matrix theory," in 6th Int. Conf. Wireless Commun. Networking and Mobile Computing, Sept. 2010, pp. $1-4$.

[23] M. Matthaiou, M.R. Mckay, P.J. Smith, and J.A. Nossek, "On the condition number distribution of complex wishart matrices," IEEE Trans. Commun., vol. 58, no. 6, pp. $1705-1717$, June 2010

[24] C. Zhong, M.R. McKay, T. Ratnarajah, and Kai-Kit Wong, "Distribution of the demmel condition number of wishart matrices," IEEE Trans. Commun., vol. 59, no. 5, pp. 1309 -1320, May 2011.

[25] A. Kortun, M. Sellathurai, T. Ratnarajah, and Caijun Zhong, "Distribution of the ratio of the largest eigenvalue to the trace of complex wishart matrices," IEEE Trans. Signal Process., vol. 60 , no. 10 , pp. $5527-5532$, oct. 2012.

[26] R. Zhang, T. Lim, Y. C. Liang, and Y. Zeng, "Multi-antenna based spectrum sensing for cognitive radios: A GLRT approach," IEEE Trans. Commun., vol. 58, no. 1, pp. $84-88$, Jan. 2010.

[27] R. Lo andpez Valcarce, G. Vazquez-Vilar, and J. Sala, "Multiantenna spectrum sensing for cognitive radio: overcoming noise uncertainty," in 2nd Int. Workshop Cognitive Inf. Process., June 2010, pp. $310-315$.

[28] D.B. Williams and D.H. Johnson, "Using the sphericity test for source detection with narrow-band passive arrays," IEEE Trans. Acoustics, Speech and Signal Process., vol. 38, no. 11, pp. 2008 -2014, nov 1990.

[29] S. Chatzinotas and B. Ottersten, "Free probability based capacity calculation of multiantenna gaussian fading channels with cochannel interference," Physical Commun., vol. 4, no. 3, pp. 206-217, 2011.

[30] S. Chatzinotas and B. Ottersten, "Interference mitigation techniques for clustered multicell joint decoding systems," EURASIP J. Wireless Commun. and Networking, vol. 2011, no. 1, pp. 1-13, 2011. 